\title{
Age-related changes in baroreflex sensitivity and cardiac autonomic tone in children mirrored by regional brain gray matter volume trajectories
}

\author{
Mark W. DiFrancesco ${ }^{1}$, Abu Shamsuzzaman ${ }^{2}$, Keith B. McConnell ${ }^{2}$, Stacey L. Ishman ${ }^{2}$, Nanhua Zhang ${ }^{3}$, Guixia Huang $^{3}$, \\ Monir Hossain ${ }^{3}$ and Raouf S. Amin ${ }^{2}$
}

BACKGROUND: The baroreflex and central autonomic brain regions together control the cardiovascular system. Baroreflex sensitivity (BRS) decreases with age in adults. Age-related changes in brain regions for cardiovascular control in children are unknown. We studied age-related changes in BRS, cardiac autonomic tone, and gray matter volume (GMV) of brain regions associated with cardiovascular control.

METHODS: Beat-to-beat blood pressure and heart rate (HR) were recorded in 49 children (6-14 years old). Spontaneous BRS was calculated by the sequence method. Cardiac autonomic tone was measured by spectral analysis of HR variability. GMV was measured using voxel-based morphometryin 112 healthy children (5-18 years old).

RESULTS: Age-related changes in BRS were significantly different in children $<10$ years and $\geq 10$ years. Age-related changes in GMV in regions of interest (ROI) were also significantly different between children $<10$ and $\geq 10$ years and between children $<11$ and $\geq 11$ years. However, agerelated changes in cardiac autonomic tone were progressive. CONCLUSIONS: Significant changes in BRS trajectories between $<10$ and $\geq 10$ years may be associated with similar age-related changes of GMV in brain ROI. This new knowledge will guide future studies examining whether childhood cardiovascular disruption manifests as deviated maturation trajectories of specific brain regions.

D uring brain maturation, neural plasticity results in age-related structural and functional changes of the brain, which include myelination and dendritic pruning of gray matter (1). Abnormalities in either maturation or neural plasticity have been linked to human diseases $(2,3)$. In the setting of neurobehavioral disorders, characteristic maturational abnormalities of specific brain regions have been identified in association with attention deficit disorders and psychiatric conditions $(4,5)$.

Similar studies of brain maturation in regions responsible for cardiovascular control have not been conducted. Thus, the role of brain maturation in autonomic control of the cardiovascular system, which evolves as children age, is not clearly understood. Furthermore, it remains unknown how deviation from the normal maturation trajectory of specific brain regions contributes to cardiovascular dysfunctions and diseases. The objectives of this study are to describe the developmental changes of the baroreflex and of brain regions linked to autonomic control in normal healthy children spanning the school-age years.

Baroreflex sensitivity (BRS) is an important indicator of cardiac autonomic regulation. Arterial baroreceptors are sensory nerve endings that innervate the walls of the large arteries; increases or decreases in arterial pressure and vascular stretch alter the frequency of action potential discharge (baroreceptor activity) transmitted along the carotid sinus and aortic depressor nerves to the nucleus tractus solitarius in the central nervous system. In addition to the afferent input from the primary baroreceptors located on the thoracic arteries, the central nervous system also continuously modulates baroreceptor-mediated autonomic tone to the heart and blood vessels $(6,7)$. Studies in animals and humans suggest that specific cortical and subcortical regions (including insula, amygdala, caudate, and cingulate) are associated with central autonomic modulation of cardiovascular function (8-13). Therefore, injuries to these brain regions are associated with autonomic dysfunction and abnormalities in blood pressure (BP) regulation (14-16).

Since structural changes in major thoracic arteries are minimal during early childhood (17) when compared to adult life, we anticipate that the BRS maturation in children may primarily be associated with development of the brain regions associated with autonomic function. Therefore, tracking BRS against norms during development, achievable by a simple and noninvasive method, may reflect early childhood maturation of the brain regions associated with cardiovascular regulation.

In this study, we characterize the age-related changes of baroreceptor function, measured as BRS, in a cohort of

\footnotetext{
${ }^{1}$ Pediatric Neuroimaging Research Consortium, Department of Radiology, Cincinnati Children's Hospital Medical Center, Cincinnati, Ohio; ${ }^{2}$ Division of Pulmonary Medicine, Cincinnati Children's Hospital Medical Center, Cincinnati, Ohio; ${ }^{3}$ Division of Biostatistics and Epidemiology, Cincinnati Children's Hospital Medical Center, Cincinnati, Ohio. Correspondence: Mark W. DiFrancesco (Mark.DiFrancesco@CCHMC.ORG) 


\section{Child age, baroreflex, and gray matter Articles}

healthy children. We then determine, in a separate cohort of healthy children, whether the same characteristic maturation trajectory is followed by gray matter volume (GMV) within specific anatomical regions known to play essential roles in cardiovascular control.

\section{METHODS}

\section{BRS Cohort}

A group of 49 children recruited at Cincinnati Children's Hospital Medical Center, with ages evenly distributed between 6.2 and 14.2 years, underwent continuous measurements of beat-to-beat BP, electrocardiogram (ECG) and respiration. A medical history and physical examination were performed in all subjects. Children were excluded if they had an acute or chronic medical disorder, including cardiovascular, cerebrovascular, or genetic conditions, or if they used any regular medications. Exclusions also included sleep disorders, including sleep-disordered breathing or alveolar hypoventilation, as determined by overnight polysomnography. Age- and genderspecific BMI- $z$ were calculated using reference data available in the Centers for Disease Control and Prevention 2000 growth charts for the United States (18). Signed informed consent and assent for children over 7 years of age were obtained from each study participant before study initiation. The study was approved by the Cincinnati Children's Hospital Medical Center Institutional Review Board.

\section{MRI Cohort}

Structural imaging data in typically developing children were provided by the National Institutes of Health-funded CMIND database (Data presented in this work was obtained from the CMIND database, provided by the Pediatric Functional Neuroimaging Research Network at https://research.cchmc.org/c-mind/. This Network and the resulting CMIND database was supported by contract from the Eunice Kennedy Shriver National Institute of Child Health and Human Development (HHSN275200900018C).) at Cincinnati Children's Hospital Medical Center. We extracted a total of 274 high-resolution structural images from this source for 112 healthy children, who were awake during scanning, with ages evenly distributed in the range spanning 5 to 18 years. A subset of 31 of these subjects participated in a longitudinal series, starting between the ages of $\sim 7$ and 9 years, with an interval of $\sim 1$ year. Twenty-one of these subjects also underwent scanning after two yearly intervals. All but 6 of the 112 subjects participated in two separate scanning sessions within a given year, each with its own structural image, with a median of 22 days between sessions (range 0-284 days). Children in the magnetic resonance imaging cohort were not screened for sleep disordered breathing.

\section{METHODS}

Spontaneous BRS. Blood pressure wave forms were recorded continuously by finger arterial plethysmography (Portapress; TNOTPD Biomedical Instrumentation, Amsterdam, Netherlands). Threelead surface ECG was recorded with Grass Telefactor System (Grass Telefactor, West Warwick, RI). The data were digitized with a sampling rate of $500 \mathrm{~Hz}$. R-waves on ECG were determined with custom software developed in our laboratory using a derivative and peak detection algorithm. The automated detection of intervals between two R-waves ( $\mathrm{R}-\mathrm{R}$ interval) was visually inspected for errors and manually edited to remove artifacts and ectopic beats. The systolic BP (SBP) was calculated as the maximum pressure on BP recordings during each cardiac cycle. Spontaneous BRS was measured during a 30-min recording while subjects were awake and laying supine in bed, using the sequence method, described in detail in our earlier study (19). In brief, the sequence method is based on the spontaneous and concurrent linear relationship between changes in SBP and R-R interval on ECG. Open loop computer analysis identified spontaneously occurring sequences of three or four heartbeats in which SBP increased by at least $1 \mathrm{~mm} \mathrm{Hg}$, and
$\mathrm{R}$-wave interval increased by at least $5 \mathrm{~ms}$. Spontaneous sequences of heartbeats in which SBP and R-wave interval decreased were also identified and analyzed separately $(20,21)$. The slope of the regression of SBP against R-wave interval describes BRS.

Cardiac Autonomic Tone by Power Spectral Analysis of HR Variability. Cardiac autonomic tone was measured by calculating spectral power in LF $(0.04-0.15 \mathrm{~Hz})$ and $\mathrm{HF}(0.15-0.5 \mathrm{~Hz})$ regimes, of beat-to-beat sequences of HR. Uniformly sampled, 300-s recordings of ECG data were smoothed by cubic spline interpolation at $2 \mathrm{~Hz}$, de-trended (using a fifth-order polynomial), and Hanning filtered. The power spectrum of each data set was calculated as the square of the Fourier transformation and integrated for LF and HF contributions and the ratio LF/HF. Details of method used for power spectral analysis of HR variability was described in our previous study (19).

Imaging Methods. All imaging data were obtained on the same 3Tesla Philips Achieva scanner (Philips Healthcare, Best, The Netherlands). The structural data extracted from CMIND were T1-weighted whole brain scans, acquired with a 32-channel SENSE head coil, with acquisition parameters: TR $8.1 \mathrm{~ms}$, TE $3.7 \mathrm{~ms}$, inversion recovery time $941 \mathrm{~ms}$, flip angle $8^{\circ}$, matrix $160 \times 224 \times 256$ voxels, and voxel size $1 \mathrm{~mm}$ isotropic (total time $5 \mathrm{~min}, 16 \mathrm{~s}$ ). Regional GMV was assessed using voxel-based morphometry analysis (22), a technique that allows for objective normalization and segmentation of high-resolution structural magnetic resonance images resulting in voxel-wise, whole-brain determination of GM and white matter tissue volume and density. This analysis was carried out using the voxel-based morphometry (VBM) 8 toolbox (23) that works in conjunction with Statistical Parametric Mapping Software SPM8 under the Matlab computing environment (The Mathworks, Natick, MA). The high-resolution structural images were first bias corrected for intensity homogeneities followed by iterative normalization to a standard MNI (Montreal Neurological Institute) template space and segmentation into GM, WM, and cerebrospinal fluid maps employing affine and nonlinear transformations using established priors for the three tissue classes (24). Normalization was refined under the DARTEL (25) high-dimensional warping routine employing the IXI550 template. A spatially adaptive nonlocal means filter (26) and a Markov random field model (27) provided further denoizing. After completion of these steps, the GM segmented maps correspond to tissue density. The Jacobian of the normalization transformation, characterizing the distortions caused by the normalization process, was applied to modulate the GM density maps resulting in a voxel-wise representation of tissue volume. We modulated the nonlinear component of the transformation, resulting in volume maps that effectively account for differences in brain volume. Finally, tissue maps were spatially smoothed via an $8 \mathrm{~mm}$ isotropic Gaussian kernel to further reduce noise and to make voxel values more normally distributed.

\section{Statistical Analysis}

Age-related changes in BRS and cardiac autonomic tone were first characterized using spline fits of various orders, selecting representative curves based on statistical significance. The best fitting curve had a single peak in BRS occurring at intermediate ages. To find an age defining a change point in BRS trajectory, a broken-stick model (28) was employed. In brief, the broken-stick model appears as: $B R S_{-}$outcome $=\beta_{0}+\beta_{1}$ age $+\beta_{2}(\text { age }-C)^{+}+\varepsilon$, where $C$ is the change point, the term $(\text { age }-C)^{+}$is zero if $($ age $-C)$ is negative and the value of $($ age $-C)$ if it is positive, $\beta_{0}$ is the intercept, $\beta_{1}$ is the slope prior to the change point, and $\beta_{2}$ the change in slope after the change point. The aim is to estimate $C$ in addition to the other parameters as a nonlinear regression fit. In addition to the broken-stick model, we also considered the random forest method for finding the age change point using the "rpart" package available in $\mathrm{R}$ statistical software (The R 


\section{Articles | DiFrancesco et al.}

Foundation for Statistical Computing, Vienna, Austria). All the statistical analyses were performed using SAS software, version 9.3 (SAS Institute, Cary, NC), and $\mathrm{R}$ version 3.2.3. The level of significance for statistical inference was set a priori at $\alpha=0.05$.

Once the age change-point was determined for the BRS data, clusters of voxels in the brain were sought with piecewise linear behavior with age with the same change-point. Since structural MRI acquisition was repeated for most subjects and for a variety of times, we calculated a mean GMV map and corresponding mean age for each of the 112 subjects. The series of mean GMV maps extracted from CMIND were fitted, voxel-wise, to a multivariate model for linear age dependence with terms for slope and intercept included separately for ages less than and greater than the change-point age, as identified for the BRS. Contrast in slope between the two age regimes was determined per voxel and a nominal threshold of $T>2.85$ was imposed. This threshold was a compromise, chosen to form clusters of voxels no larger than the typical anatomical ROI, yet large enough to reach significance at the cluster level. Suprathreshold clusters of voxels, restricted to specific anatomical ROI, were assessed for statistical significance at $P<0.05$, family-wise error corrected for multiple comparisons within each ROI. Cortical and subcortical ROI associated with autonomic and cardiovascular functions were selected based on previous studies in animal models and humans. A complete list of the 40 ROIs considered in our analysis is provided in Table 1. Anatomical ROI masks were extracted from the Automatic Anatomical Labeling Atlas stored in the WFU Pick Atlas toolbox under SPM8.

Table 1. Brain regions of interest related to cardiovascular control

\section{Brain region of interest}

Midbrain, bilateral (1-3)

Pons, bilateral $(1,3,4)$

Medulla, bilateral $(1,4)$

Thalamus $(2,3)$

Hypothalamus, bilateral $(3,5)$

Cerebellum, anterior, bilateral $(2,3,5,6)$

Cerebellum, posterior, bilateral $(2,3,5,6)$

Amygdale $(1,2,4)$

Hippocampus (3)

Caudate (3)

Putamen $(7,8)$

Insula, entire (2,4-6)

Insula, anterior half (1)

Insula, posterior half $(3,6)$

Cingulate gyrus, anterior $(1,2,4,6)$

Cingulate gyrus, posterior (5)

Frontal gyrus, middle $(2,6)$

Frontal gyrus, medial orbital (6)

Frontal gyrus, inferior orbital (4)

Frontal gyrus, inferior opercular (2)

Precentral gyrus $(2,6)$

Postcentral gyrus (2)

Precuneus $(2,5)$

All brain areas listed are split into separate left and right hemisphere regions, unless otherwise indicated.

\section{RESULTS}

\section{Characteristics of Study Population}

Age-related changes in BRS were measured in 49 healthy children with average age of $10.1 \pm 2.1$ years with an age range from 6.2 to 14.2 years. Among these subjects, $47 \%$ were girls and $75 \%$ were Caucasians. Average body mass index (BMI) and $Z$-scores for BMI (BMI- $z$ ) were $18.1 \pm 3.6$ and $0.2 \pm 1 \mathrm{~kg} /$ $\mathrm{m}^{2}$, respectively. Average systolic and diastolic BP were $93 \pm 19$ and $47 \pm 15 \mathrm{~mm} \mathrm{Hg}$ respectively.

In the Cincinnati MR Imaging of NeuroDevelopment (CMIND) population, among 112 healthy children, $49 \%$ were girls. Average age was $10.9 \pm 3.8$ years with an age range from 5.2 to 18.5 years. Average BMI and BMI- $z$ were $18.9 \pm 3.4$ and $0.3 \pm 0.9 \mathrm{~kg} / \mathrm{m}^{2}$, respectively. Among these children, $67 \%$ were Caucasian.

Demographically, the BRS and CMIND groups of children did not differ significantly with respect to age $(P=0.1)$, BMI $(P=0.2)$, BMI $z(P=0.3)$, gender $(P=0.8)$, or race $(P=0.3)$.

\section{Age-Related Changes in BRS}

Spline curves providing the best fit of the BRS data showed that around the age of 10 years there was a change of BRS maturation trajectory for both increasing and decreasing BP. Broken-stick modeling applied to BRS data for increasing and decreasing BP resulted in change-points at 9.7 years $(95 \%$ confidence interval $(\mathrm{CI}): 7.7,11.6)$ and 10.0 years $(95 \% \mathrm{CI}$ : $8.3,11.8)$, respectively. The random forest method yielded change-point values of 10.7 and 10.9 years, respectively. Consequently, we considered both 10 and 11 years as changepoint ages in subsequent statistical analyses.

Age-related changes in spontaneous BRS for increasing $(P=0.03)$ and decreasing BP $(P=0.02)$ were significantly different between children $<10$ and $\geq 10$ years of age (Figure 1). Spontaneous BRS for increasing and decreasing BP progressively increased with age in children $<10$ years of age and decreased with age beyond 10 years. For the changepoint at age 11 years, the results are not significant: agerelated changes in spontaneous BRS for increasing $(P=0.09)$ and decreasing BP $(P=0.06)$ were not significantly different between children $<11$ and $\geq 11$ years of age.

\section{Age-Related Changes in Cardiac Autonomic Tone}

The spline curves of age-related changes in cardiac autonomic tone did not show any significant difference in maturation trajectories around the age of 10 years or at any other age.

High-frequency (HF) and low-frequency (LF) power of HR variability progressively decreased with age (Figure 2 ) with no significant change in slope. LF/HF ratios progressively increased with age with no significant change in rate at any age. Unlike spontaneous BRS, no change-points could be identified for the trajectories of age-related changes in HF power, LF power, or the LF/HF ratio. 

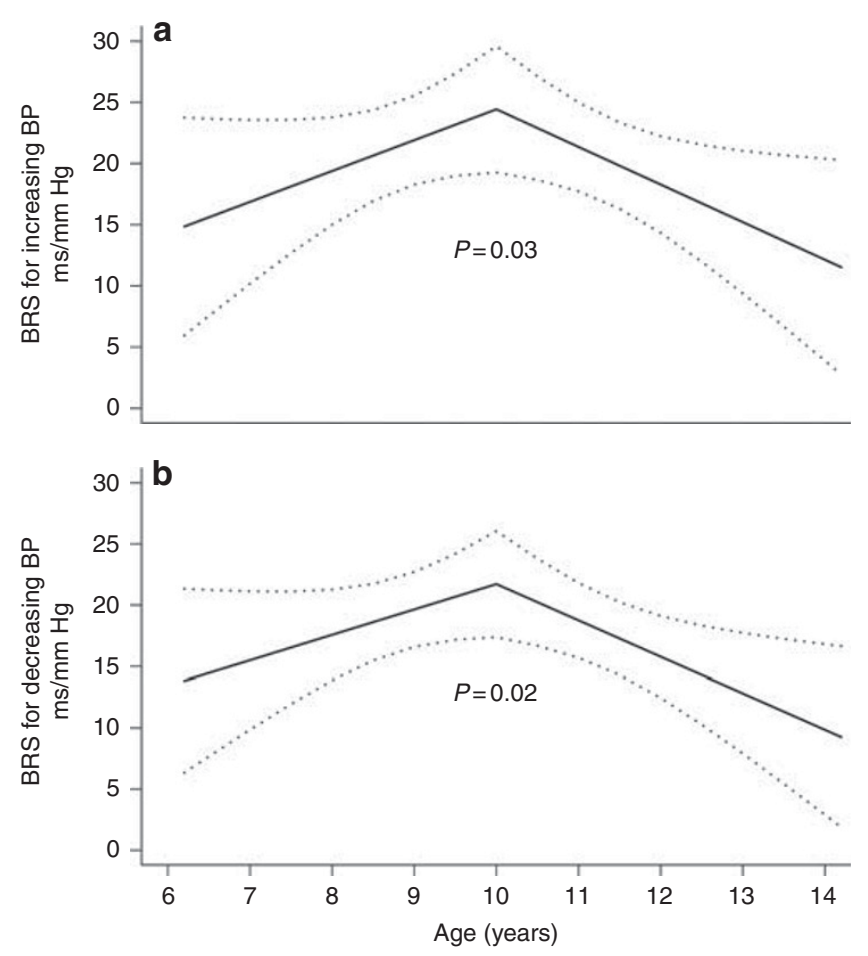

Figure 1. Age-related changes in baroreflex sensitivity (BRS) for (a) increasing and (b) decreasing blood pressure (BP) with $95 \%$ confidence intervals. Age-related changes in BRS were significantly different between children $<10$ and $\geq 10$ years. BRS progressively increased with age in children $<10$ years and reduced in children $\geq 10$ years.

\section{Age-Related Changes in GMV in Brain Regions Associated with Cardiovascular Function}

Based on the regression analyses of spontaneous BRS, both 10 and 11 years of age were identified as change-points for analyzing age-related changes in GMV. The extension of the age range to 18.5 years, compared with the BRS cohort, was considered justifiable given the mid-range BRS age changepoints and the aim of comparing age-related trends rather than population means between cohorts. Age-related changes in GMV in specific ROI of the brain associated with autonomic and cardiovascular regulation were significantly different between children $<10$ and $\geq 10$ years of age (Table 2) as well as for children $<11$ and $\geq 11$ years of age (Table 3). Brain images showing regional clusters of voxels within these ROI with significant age-related changes in GMV trajectories are shown in Figures 3 and 4 for the 10- and 11year change-points, respectively. Significant clusters were found in a number of the select brain ROIs, including the cingulate, amygdala, hypothalamus, mid-brain, and putamen (Tables 2 and 3). Several areas of the brain, including the caudate, left posterior insula, right hippocampus, and medial orbital frontal gyrus had significant age-related changes in GMV only for the 11-year change-point (Table 3), while the pons was found significant only for the 10-year change-point. Examples of the maturation trajectories of GMV with significant slope changes in the left posterior insula, left
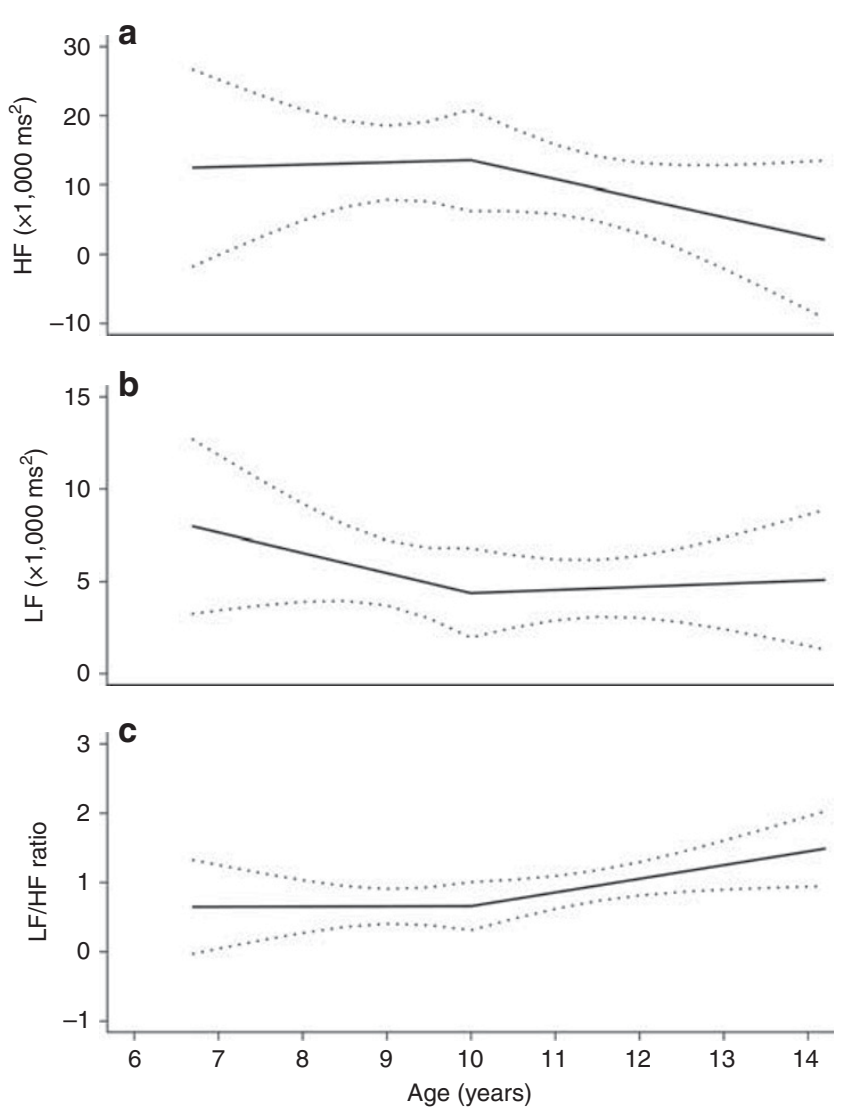

Figure 2. Age-related changes in cardiac autonomic tone for (a) lowfrequency (LF) and (b) high-frequency (HF) regimes and the (c) LF/HF ratio. LF and HF outcomes progressively decreased with age, while LF/ HF increased with age, without significant change in slope at 10 years.

putamen, right amygdala, and right hippocampus are displayed in Figure 5a-d, respectively. GMV monotonically decreased with age in the insula, amygdale, and hippocampus despite the change in slope. Conversely, in the putamen (Figure 5b), the GMV undergoes a change in slope with age from positive to negative at the change-point.

\section{DISCUSSION}

In this study, we demonstrated in two different cohorts that the age-related changes of the baroreflex and the structure of brain regions which control autonomic functions follow similar trajectories with a point of inflection occurring around the age of 10-11 years. These observations provide important baseline knowledge regarding normal development of central cardiovascular autonomic control and set the stage for future investigation of the developmental impact of disease states on GMV and autonomic control.

The trajectory of age-related changes in BRS parameters for children between the ages of 6 and 14 years increases until the age of 10 or 11 years, depending on the model, at which point BRS begins to decline with increasing age. Piecewise regression provided the age at which the BRS trajectory changed significantly. The spectral measures, describing 
Articles | DiFrancesco et al.

Table 2. List of the brain regions with age-related GMV changes in children $<10$ and $\geq 10$ years $(P<0.1)$

\begin{tabular}{|c|c|c|c|c|c|c|c|c|c|c|c|}
\hline \multirow[t]{3}{*}{ Anatomic region } & \multirow[t]{3}{*}{ Side } & \multicolumn{5}{|c|}{ Greater for $\geq 10$ years than $<10$ years } & \multicolumn{5}{|c|}{ Greater for $<10$ years than $\geq 10$ years } \\
\hline & & \multirow[t]{2}{*}{$\mathrm{P}$ value } & \multirow[t]{2}{*}{ Cluster size } & \multicolumn{3}{|c|}{ MNI coordinates $(\mathrm{mm})$} & \multirow[t]{2}{*}{$\mathrm{P}$ value } & \multirow[t]{2}{*}{ Cluster size } & \multicolumn{3}{|c|}{ MNI coordinates (mm) } \\
\hline & & & & $x$ & $y$ & $z$ & & & $x$ & $y$ & z \\
\hline Cingulate, Post & $L$ & 0.089 & 17 & -3 & -40 & 6 & & & & & \\
\hline Amygdala & $\mathrm{R}$ & 0.023 & 176 & 23 & 5 & -15 & & & & & \\
\hline Midbrain & & 0.001 & 1,728 & 12 & -16 & -12 & & & & & \\
\hline Pons & & 0.065 & 204 & -3 & -33 & -36 & & & & & \\
\hline Putamen & $\mathrm{L}$ & & & & & & 0.034 & 286 & -24 & -2 & 3 \\
\hline Putamen & $\mathrm{R}$ & 0.099 & 70 & 15 & 3 & -9 & 0.042 & 238 & 27 & -7 & 6 \\
\hline Hypothalamus & & 0.019 & 117 & -5 & 0 & -6 & & & & & \\
\hline
\end{tabular}

GMV, gray matter volume; L, left; MNI, Montreal Neurological Institute; Post, posterior; R, right.

Table 3. List of the brain regions with age-related GMV changes in children $<11$ and $\geq 11$ years $(P<0.1)$

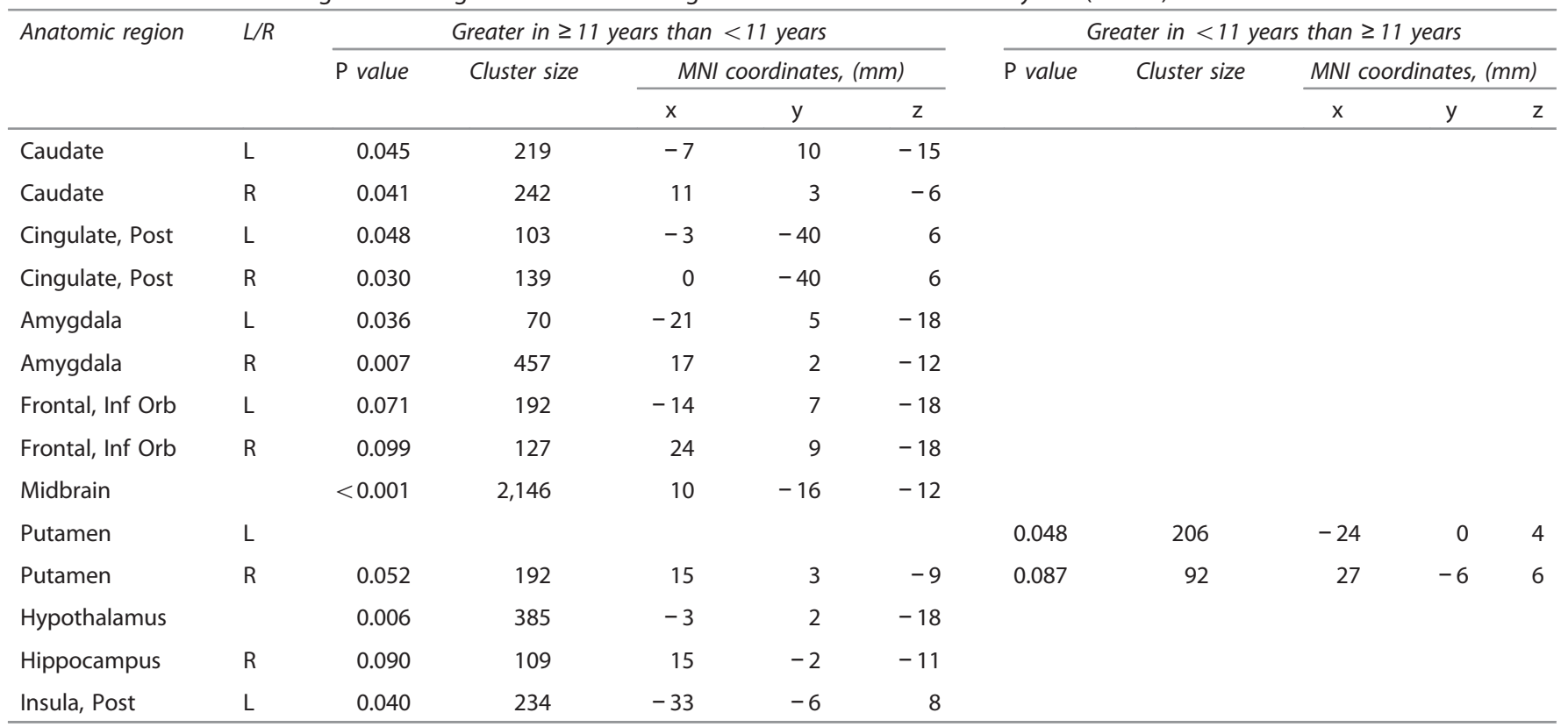

GMV, gray matter volume; Inf, inferior; L, left; MNI, Montreal Neurological Institute; Orb, orbital; Post, posterior; R, right.

sympathovagal balance, changed monotonically with age with no statistically significant change in slope for LF, HF, or LF/HF. A progressive reduction in HF variability (a measure of cardiovagal tone) and a corresponding increase in LF/HF ratio (a measure of sympathovagal balance) suggest a progressive increase in the ratio of sympathetic to parasympathetic tone. Furthermore, as shown in Figure 2, there is a change in the trajectory of the slope at the age of 10 years whereby HF continues to decline with age, while LF remains at a plateau. Although not statistically significant, it is likely that the decrease in HF, a measure reflecting parasympathetic tone, contributes in part to the change in BRS observed at age 10 years.

The arterial baroreflex is a critical mechanism for BP regulation and reduced BRS is associated with increased risk for cardiovascular morbidity and mortality (29-31). Previous adult studies have reported progressive reductions of the BRS with age $(32,33)$. Age-related reductions of BRS in adults are primarily associated with progressive changes in vessel wall thickness that alter arterial distensibility and thus pressure transduction to baroreceptors located in the wall of major thoracic arteries $(34,35)$. Although we did not examine the stiffness of thoracic arteries, it is unlikely that there is enough change in arterial wall thickness occurring at around 10 years of age to explain the marked decline in BRS seen in our study.

In adults, age-related increase in sympathetic drive has been measured by noninvasive power spectral analysis of heart rate (HR) variability and by direct recording of sympathetic nerve traffic from peripheral nerves (36-39). One acknowledged contributing factor to the progressive increase in sympathetic tone with age is the known reduction of the inhibitory effect of arterial baroreceptors on sympathetic neurons located in the brain stem as the BRS decreases with age $(34,35,40)$. However, a similar mechanism in children for progressive increases in sympathetic tone with age has yet to be investigated. We postulate that maturation and neural 


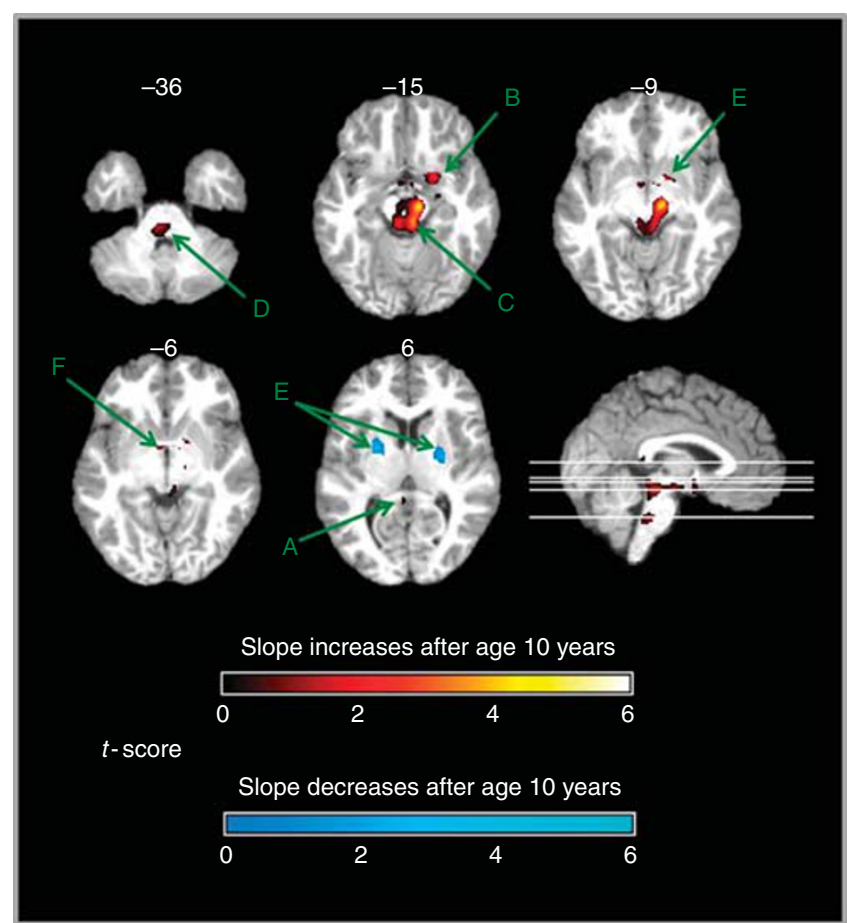

Figure 3. Clusters of voxels with significant change in gray matter volume with age in children $<10$ and $\geq 10$ years of age. Hot colors indicate increased and cool colors indicate decreased age-related changes in children $\geq 10$ years. Clusters are restricted to regions of the brain associated with autonomic and cardiovascular functions including posterior (A) cingulate, (B) amygdala, (C) midbrain, (D) pons, (E) putamen, and (F) hypothalamus. Montreal Neurological Institute (MNI) coordinate for each brain slice is shown above each image and slice locations are indicated in the sagittal view. Axial slices are shown in neurological convention (right side of brain $=$ right side of image). Clusters are significant at $P<0.1$, family-wise error corrected, after thresholding at nominal $T>2.85$.

plasticity of the central autonomic brain regions may play a critical mechanistic role in age-related changes in cardiac autonomic tone in children.

In a separate cohort of typically developing children who had undergone structural brain imaging, we used a similar piecewise regression approach to determine whether gray matter structures within select ROI associated with autonomic cardiovascular control have age-related changes similar to those observed for BRS. We found that for many of the select brain ROI, the trajectory of age-related changes in GMV changed at the ages of 10 and 11 years. These collective results demonstrate that in two different populations of healthy children, the baroreflex and cardiovascular brain regions, respectively, undergo similar functional and structural changes within overlapping age ranges.

During childhood there is major structural and functional maturation of the brain (1), progressing by ongoing myelination and dendritic pruning. Age-related neuronal proliferation, rewiring, and dendritic pruning are all critical for the development of neuronal plasticity for both motor skills and cognitive function development (41), but may also be critical for the regulation of autonomic function. Our study

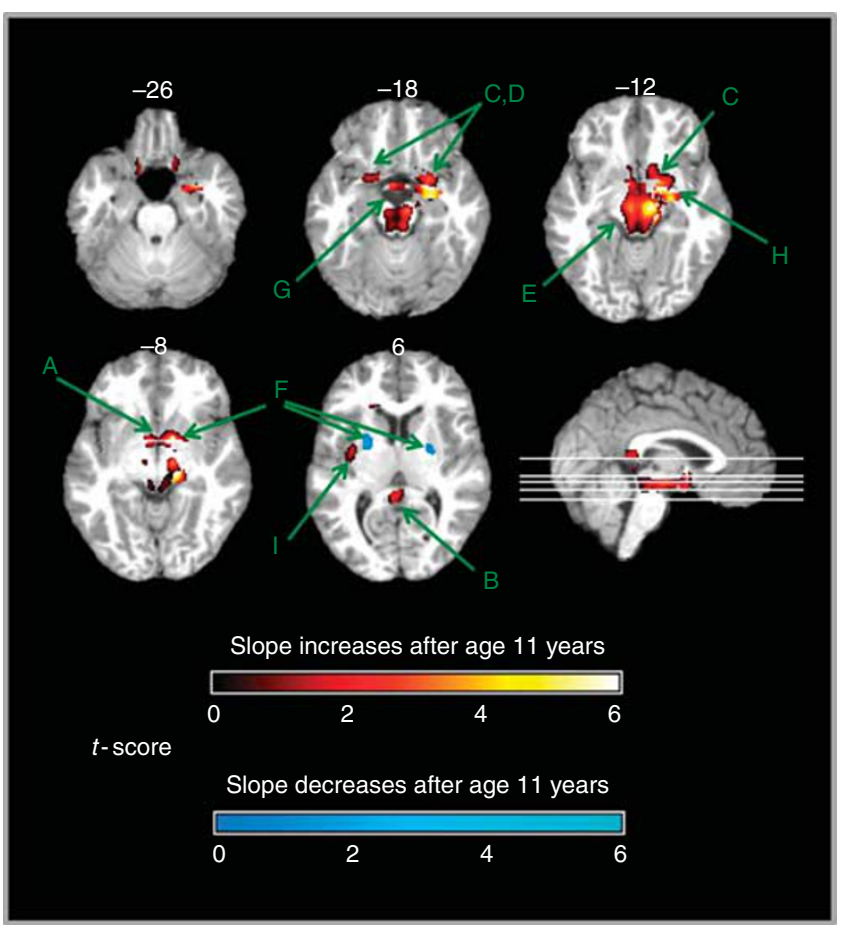

Figure 4. Clusters of voxels with significant change in gray matter volume with age in children $<11$ and $\geq 11$ years. Hot colors indicate increased and cool colors indicate decreased age-related changes in children $\geq 11$ years. Clusters are significant at $P<0.1$, family-wise error corrected, after thresholding at nominal $T>2.85$, restricted to regions of interest (ROI) known for essential role in cardiovascular regulation. Clusters are labeled as follows: (A) caudate, (B) posterior cingulate, (C) amygdala, (D) inferior orbital frontal, (E) midbrain (e), (F) putamen, (G) hypothalamus, $(\mathrm{H})$ right hippocampus, (I) and left posterior insula. Montreal Neurological Institute (MNI) coordinate for each brain slice is shown above each image and slice locations are indicated in the sagittal view. Axial slices are shown in neurological convention (right side of brain = right side of image).

is the first to demonstrate that changes in GMV trajectories with age in regions of the brain associated with autonomic and cardiovascular functions mimic maturational changes in BRS. Rates of GMV changes with age differed significantly between children older and younger than change-points at 10 or 11 years in a number of brain regions associated with cardiovascular functions including insula, cingulate, caudate, amygdala, hypothalamus, mid-brain, and pons.

A strength of the current study is the statistical exploratory approach that we adopted for two independent cohorts of healthy children; in one population we described a changepoint of age-related functional changes of autonomic control and then sought, within the other group, age-related structural changes within critical brain regions with the same change-point. Another important strength is the large sample size from the CMIND database to measure age-related changes in GMV of the brain regions associated with autonomic and cardiovascular function. Additionally, the BRS was measured in a population of healthy control subjects who were studied with clinical assessment, personal and 


\section{Articles | DiFrancesco et al.}
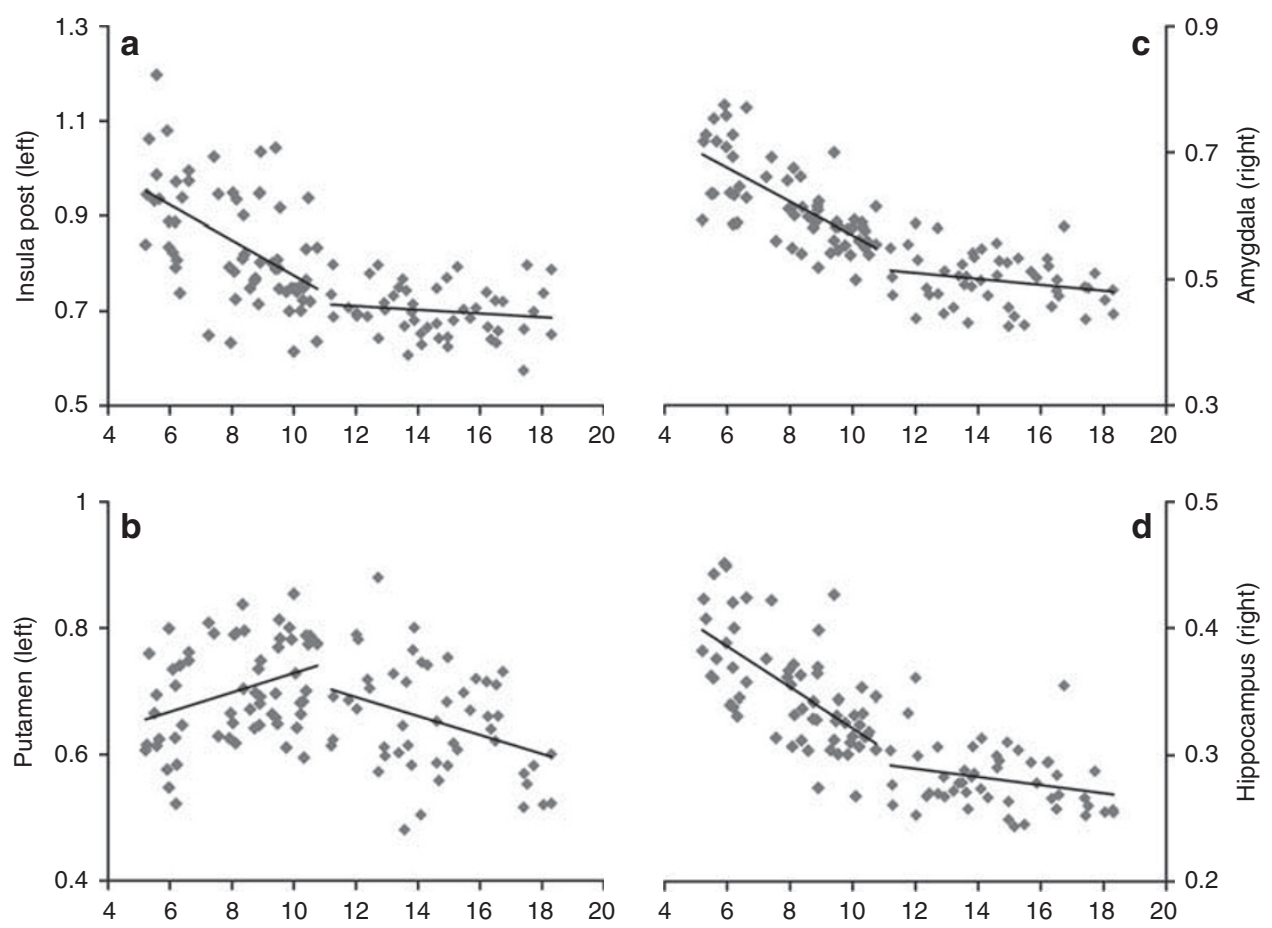

Figure 5. Age-related changes in gray matter volume (GMV) in (a) left posterior insula, (b) left putamen, (c) right amygdala, and (d) right hippocampus. Note that age-related changes in GMV shown are for the voxel of peak significance in each region for the difference between children $<11$ and $\geq 11$ years.

family medical history, physical examination, and routine investigation for diagnosis of common diseases including diabetes, cardiovascular, and renal disorders.

While this study of two different populations has its advantages, it also represents a limitation since we were unable to establish a direct relationship between the BRS and structural changes in the brain. We anticipate that the agerelated maturation trajectories for BRS and brain GMV reported in the current study will be similar in both populations of healthy control subjects; this is not yet proven. An additional limitation is the lack of information about the pubertal status of the children evaluated for the BRS and the imaging studies. Therefore, this study could not determine the effects of puberty onset on changes in maturation trajectories for BRS and brain volume. It is not known whether the early onset of puberty in girls affects maturation trajectories for BRS and/or brain structure. However, we did perform the appropriate statistical adjustments to investigate the effect of gender on the BRS measures. An additional limitation includes the cross-sectional study design for both the BRS and imaging studies. A longitudinal and long-term follow-up study will provide a better understanding of BRS and brain maturation in children.

In conclusion, we found that maturation trajectories for spontaneous BRS significantly changed in children for a change-point of 10 years and had changes of trending significance for a change-point at 11 years. Regionally, GMV trajectories changed significantly for change-points at both 10 and 11 years. However, progressive age-related changes in cardiac autonomic tone were not found to undergo a significant change in slope up to the age of 14 years. Additionally, noninvasive and simple measures of spontaneous BRS and cardiac autonomic functions in children may provide critical information on the structural and functional maturation of the brain autonomic regions. These cardiovascular measurements can be used as simple and inexpensive markers for assessment of childhood development of the brain and autonomic neural plasticity. Future studies will determine whether diseases suffered during childhood with potential impact on brain health, such as obstructive sleep apnea or type 2 diabetes, have significant impact on the maturation of BP regulatory mechanisms and development of cardiovascular morbidity and mortality.

\section{STATEMENT OF FINANCIAL SUPPORT}

This study was supported by a National Institutes of Health grant (R01 HL080670) and Clinical Translational Research Center (MO1RR 08084-08), Cincinnati Children's Hospital.

Disclosure: The authors declare no conflict of interest.

\section{REFERENCES}

1. Giedd JN, Blumenthal J, Jeffries NO, et al. Brain development during childhood and adolescence: a longitudinal MRI study. Nat Neurosci 1999;2:861-3.

2. Luby JL, Belden AC, Jackson JJ, et al. Early childhood depression and alterations in the trajectory of gray matter maturation in middle childhood and early adolescence. JAMA Psychiatry 2016;73:31-8.

3. Walhovd KB, Tamnes CK, Fjell AM. Brain structural maturation and the foundations of cognitive behavioral development. Curr Opin Neurol 2014;27:176-84. 


\section{Child age, baroreflex, and gray matter Articles}

4. Thillay A, Roux S, Gissot V, et al. Sustained attention and prediction: distinct brain maturation trajectories during adolescence. Front Hum Neurosci 2015;9:519.

5. McLaughlin KA, Fox NA, Zeanah CH, Sheridan MA, Marshall P, Nelson CA. Delayed maturation in brain electrical activity partially explains the association between early environmental deprivation and symptoms of attention-deficit/hyperactivity disorder. Biol Psychiatry 2010;68:329-36.

6. Matsukawa K. Central command: control of cardiac sympathetic and vagal efferent nerve activity and the arterial baroreflex during spontaneous motor behaviour in animals. Exp Physiol 2012;97:20-8.

7. Ogoh S, Wasmund WL, Keller DM, et al. Role of central command in carotid baroreflex resetting in humans during static exercise. J Physiol 2002;543:349-64.

8. Kimmerly DS, O'Leary DD, Menon RS, Gati JS, Shoemaker JK. Cortical regions associated with autonomic cardiovascular regulation during lower body negative pressure in humans. J Physiol 2005;569:331-45.

9. Napadow V, Dhond R, Conti G, Makris N, Brown EN, Barbieri R. Brain correlates of autonomic modulation: combining heart rate variability with fMRI. Neuroimage 2008;42:169-77.

10. Nagai M, Hoshide S, Kario K. The insular cortex and cardiovascular system: a new insight into the brain-heart axis. J Am Soc Hypertens 2010;4:174-82.

11. James C, Macefield VG, Henderson LA. Real-time imaging of cortical and subcortical control of muscle sympathetic nerve activity in awake human subjects. Neuroimage 2013;70:59-65.

12. Kimmerly DS, Morris BL, Floras JS. Apnea-induced cortical BOLD-fMRI and peripheral sympathoneural firing response patterns of awake healthy humans. PLoS ONE 2013;8:e82525.

13. Thayer JF, Ahs F, Fredrikson M, Sollers JJ III, Wager TD. A meta-analysis of heart rate variability and neuroimaging studies: implications for heart rate variability as a marker of stress and health. Neurosci Biobehav Rev 2012;36:747-56.

14. Goldstein B, Kempski MH, DeKing D, et al. Autonomic control of heart rate after brain injury in children. Crit Care Med 1996;24:234-40.

15. Kallmunzer B, Breuer L, Kahl N, et al. Serious cardiac arrhythmias after stroke: incidence, time course, and predictors-a systematic, prospective analysis. Stroke 2012;43:2892-7.

16. Takahashi C, Hinson HE, Baguley IJ. Autonomic dysfunction syndromes after acute brain injury. Handb Clin Neurol 2015;128:539-51.

17. Lenard Z, Studinger P, Mersich B, Kocsis L, Kollai M. Maturation of cardiovagal autonomic function from childhood to young adult age. Circulation 2004;110:2307-12.

18. Ogden CL, Kuczmarski RJ, Flegal KM, et al. Centers for Disease Control and Prevention 2000 growth charts for the United States: Improvements to the 1977 National Center for Health Statistics version. Pediatrics 2002;109:45-60.

19. McConnell K, Somers VK, Kimball T, et al. Baroreflex gain in children with obstructive sleep apnea. Am J Respir Crit Care Med 2009;180:42-8.

20. Bertinieri G, Di Rienzo M, Cavallazzi A, Ferrari AU, Pedotti A, Mancia G. Evaluation of baroreceptor reflex by blood pressure monitoring in unanesthetized cats. Am J Physiol 1988;254:H377-83.

21. Legramante JM, Marciani MG, Placidi F, et al. Sleep-related changes in baroreflex sensitivity and cardiovascular autonomic modulation. J Hypertens 2003;21:1555-61.
22. Ashburner J, Friston KJ. Voxel-based morphometry-the methods. Neuroimage 2000;11:805-21.

23. Gaser C VBM8. Jena, Germany: University of Jena.

24. Ashburner J, Friston KJ. Unified segmentation. Neuroimage 2005;26:839-51.

25. Ashburner J. A fast diffeomorphic image registration algorithm. Neuroimage 2007;38:95-113.

26. Manjon JV, Coupe P, Marti-Bonmati L, Collins DL, Robles M. Adaptive non-local means denoising of MR images with spatially varying noise levels. J Magn Reson Imag 2010;31:192-203.

27. Rajapakse JC, Giedd JN, Rapoport JL. Statistical approach to segmentation of single-channel cerebral MR images. IEEE Trans Med Imaging 1997;16:176-86.

28. Toms JD, Lesperance ML. Piecewise regression: a tool for identifying ecological thresholds. Ecology 2003;84:2034-41.

29. Garcia R, Sosner P, Laude D, Hadjadj S, Herpin D, Ragot S. Spontaneous baroreflex sensitivity measured early after acute myocardial infarction is an independent predictor of cardiovascular mortality: results from a 12year follow-up study. Int J Cardiol 2014;177:120-2.

30. De Ferrari GM, Sanzo A, Bertoletti A, Specchia G, Vanoli E, Schwartz PJ. Baroreflex sensitivity predicts long-term cardiovascular mortality after myocardial infarction even in patients with preserved left ventricular function. J Am Coll Cardiol 2007;50:2285-90.

31. Johansson M, Gao SA, Friberg P, et al. Baroreflex effectiveness index and baroreflex sensitivity predict all-cause mortality and sudden death in hypertensive patients with chronic renal failure. J Hypertens 2007;25:163-8.

32. Monahan KD. Effect of aging on baroreflex function in humans. Am J Physiol Regul Integr Comp Physiol 2007;293:R3-12.

33. Piccirillo G, Di Giuseppe V, Nocco M, et al. Influence of aging and other cardiovascular risk factors on baroreflex sensitivity. J Am Geriatr Soc 2001;49:1059-65.

34. Monahan KD, Dinenno FA, Seals DR, Clevenger CM, Desouza CA, Tanaka H. Age-associated changes in cardiovagal baroreflex sensitivity are related to central arterial compliance. Am J Physiol Heart Circ Physiol 2001;281:H284-9.

35. Dinenno FA, Jones PP, Seals DR, Tanaka H. Age-associated arterial wall thickening is related to elevations in sympathetic activity in healthy humans. Am J Physiol Heart Circ Physiol 2000;278:H1205-10.

36. Piccirillo G, Cacciafesta M, Viola E, et al. Influence of aging on cardiac baroreflex sensitivity determined non-invasively by power spectral analysis. Clin Sci (Lond) 2001;100:267-74.

37. Ramaekers D, Ector H, Aubert AE. The influence of age and gender on heart rate variability (HRV). J Am Coll Cardiol 1999;33:900-2.

38. Almeida-Santos MA, Barreto-Filho JA, Oliveira JL, Reis FP, da Cunha Oliveira CC, Sousa AC. Aging, heart rate variability and patterns of autonomic regulation of the heart. Arch Gerontol Geriatr 2016;63:1-8.

39. Matsukawa T, Sugiyama Y, Mano T. Age-related changes in baroreflex control of heart rate and sympathetic nerve activity in healthy humans. J Auton Nerv Syst 1996;60:209-12.

40. Huang CC, Sandroni P, Sletten DM, Weigand SD, Low PA. Effect of age on adrenergic and vagal baroreflex sensitivity in normal subjects. Muscle Nerve 2007;36:637-42.

41. Baird AA, Gruber SA, Fein DA, et al. Functional magnetic resonance imaging of facial affect recognition in children and adolescents. J Am Acad Child Adolesc Psychiatry 1999;38:195-9. 